\title{
Expression of dual angiogenic/neurogenic growth factors in human primary brain tumors
}

\author{
Maud Clemessy $\cdot$ Robert C. Janzer • \\ Benoit Lhermitte $\cdot$ Jean-Marie Gasc $\cdot$ \\ Lucienne Juillerat-Jeanneret
}

Received: 22 December 2010/Accepted: 16 September 2011/Published online: 7 October 2011

(C) Springer Science+Business Media, LLC. 2011

\begin{abstract}
Brain tumors, benign or malignant, are characterized by a very high degree of vascularization. Recent accumulating evidence suggests that during development the neuronal wiring follows the same routes as the vasculature and that these two systems may share some of the same factors for guidance. Thus, expression of dual angiogenic/neurogenic growth factors was evaluated by in situ hybridization in human primary brain tumors of three different types, i.e., astrocytomas, oligodendrogliomas, and ependymomas, of increasing grades, in relation with the grade and type of the tumor. For this evaluation we selected vascular endothelial growth factor (VEGF-A) and its receptors VEGF-R1 and VEGF-R2 and the neuropilins 1 and 2 (NRP-1 and NRP-2), which have proangiogenic properties, platelet-derived growth factor (PDGF) receptor-beta (PDGF-R $\beta$ ), which is required for the functional maturation of blood vessels, the ephrins and their Eph receptors, angiotensinogen (AGT) and thrombospondin-2 (TSP-2), which have potential antiangiogenic properties, and netrin-1 (Net-1), which regulates vascular architecture. We show that the expression of the VEGF-
\end{abstract}

This manuscript is dedicated to Robert-Charles Janzer, who died suddenly on September 27th, 2010.

M. Clemessy · J.-M. Gasc

INSERM U833, Collège-de-France, 11 place Marcelin Berthelot, Paris, France

R. C. Janzer · B. Lhermitte · L. Juillerat-Jeanneret Centre Hospitalier Universitaire Vaudois (CHUV) and

University of Lausanne (UNIL), Lausanne, Switzerland

L. Juillerat-Jeanneret $(\bowtie)$

University Institute of Pathology, Rue du Bugnon 25,

1011 Lausanne, Switzerland

e-mail: lucienne.juillerat@chuv.ch
NRP system, PDGF-R $\beta$, TSP-2, AGT, and Net-1 are differentially regulated, either increased or decreased, in relation with the type and grade of the tumor, whereas regulation of the ephrinB system does not seem to be relevant in these human brain tumors.

Keywords Human astrocytoma - Human glioblastoma . Human ependymoma - Human oligodendroglioma .

VEGF · Ephrin · Netrin · Neuropilin · Angiotensinogen · Thrombospondin $\cdot \operatorname{PDGFR} \beta$

$\begin{array}{ll}\text { Abbreviations } \\ \text { VEGF } & \text { Vascular endothelial growth factor } \\ \text { NRP } & \text { Neuropilin } \\ \text { PDGFR } \beta & \text { Platelet-derived growth factor receptor-beta } \\ \text { Eph } & \text { Ephrin } \\ \text { AGT } & \text { Angiotensinogen } \\ \text { TSP-2 } & \text { Thrombospondin-2 } \\ \text { Net-1 } & \text { Netrin-1 } \\ \text { Astro } & \text { Astrocytoma } \\ \text { GBM } & \text { Glioblastoma multiforme } \\ \text { Oligo } & \text { Oligodendroglioma } \\ \text { Epend } & \text { Ependymoma }\end{array}$

Introduction

A histological feature common to all brain tumors is a high density of vascularization, required for the rapid growth of the tumor. It is generally accepted that cancer recapitulates, at least in part, developmental programs. Normal vasculature is hierarchically assembled, while the blood vessels of cancers retain the disordered and leaky structure of immature 
developing vasculature. The status of the specialized cerebral vasculature, the blood-brain barrier (BBB), is modified in brain tumors, with increased permeability in high-grade cancer (grade III and IV tumors).

Extensive similarities have been recognized between the development of nerves and blood vessels. Branched structures require guidance cues, and it has been shown that during development these systems share the same molecular cues [1-3]. The build-up of a vascular system requires precise control of the growth and branching of new vessels. The genetic control of families of molecules, in particular of peptides and their cognate receptors, is responsible for attractive and repulsive cues. Some of these peptide pathways have also been previously involved in controlling neuronal guidance, modulating physiological as well as pathological processes. Thus, the overall similarity observed between the vascular tree and the nervous system has led to the concept of two integrated systems, possibly using the molecular control of the same factors. These neural/vascular families of peptides have been evaluated in detail in vascular development, however they have received less attention, with the exception of the vascular endothelial growth factor (VEGF) system, in postdevelopmental stage of angiogenesis in humans, in particular in angiogenic neovessels associated to human primary cancers of the central nervous system [4]. Therefore, cross-reactivity between growth factors regulating the development of the central nervous system (CNS) and angiogenesis, including tumoral angiogenesis, and dual functions for these factors, may be postulated.

We have previously shown that the components of the renin-angiotensin system may be survival factors for human brain-derived tumors [5]. On the other hand, in the nervous system the ephrin and NRP-VEGF systems exert repulsive action on axonal guidance, the netrins are involved in neuron migration, and during neural tube development, netrin-1 antagonizes platelet-derived growth factor (PDGF) effects. In the present study, the parallelism between the vascular tree and the neural architecture together with the concept of factors targeting both blood vessels and nerves led us to assess using molecular histology the expression of such dual neurogenic/angiogenic factors in primary human glial and nonglial brain tumors of different grades. For this evaluation we selected VEGF-A and its receptors VEGF-R1 and VEGF-R2 as well as neuropilins 1 and 2 (NRP-1 and NRP-2), which have proangiogenic properties, platelet-derived growth factor receptor-beta (PDGF-R $\beta$ ), which is required for the functional maturation of blood vessels, netrin-1 (Net-1), which regulates vascular architecture, and the ephrinBs and their Eph receptors, angiotensinogen (AGT) and thrombospondin-2 (TSP-2), which have potential antiangiogenic properties. Our aims are to decipher putative cooperation between these dual vascular/neural growth factors and to evaluate new potential markers which can be associated with brain cancer progression and may be targets for therapeutic intervention.

\section{Materials and methods}

Ex vivo collection and classification of the human surgical specimens

Human brain cancer specimens $(n=6 /$ cancer type and grade) were retrospectively selected from the tissue bank of the University Institute of Pathology, according to type and grade as the selection criteria, after full diagnostic procedure had been performed. The protocol was approved by the Ethics Committee of the University Hospital (CHUV) in Lausanne. A histological hematoxylin-eosin-stained slide of the selected sample, or of the selected fragment if several tumor fragments were available, was examined by an experienced neuropathologist to control the tumoral characteristics (type and grade) of the sample, before in situ hybridization was performed. Tumors of the central nervous system were classified by the World Health Organization (WHO) classification system according to a "malignancy scale" depending on defined criteria. Grade I lesions include tumors with low proliferative potential and the possibility of cure following surgical resection only. Grade II lesions are infiltrative in nature and recur despite low proliferative activity, and may progress to higher grade of malignancy. Grade III lesions are tumors with histological evidence of malignancy, including nuclear atypia and high proliferative activity. Grade IV lesions are cytologically malignant, very highly proliferative, angiogenic and infiltrative, necrotic tumors. The WHO classification system was followed for evaluation of the selected tumors of this project. Tissues were fixed in buffered paraformaldehyde and embedded in paraffin. Paraffin-embedded tissues were cut at $5 \mu \mathrm{m}$ and deparaffinized in graded alcohols. Samples were then exposed to probes of the factors under evaluation.

\section{Molecular histology}

Sense and antisense probes were generated from all complementary DNAs (cDNAs) as control for specificity of labeling and as specific labeling probe, respectively. In situ hybridization was performed using the sense and antisense probes, according to the technique previously described in detail [6] with minor modifications. Briefly, complementary RNA (cRNA) probes were labeled by in vitro transcription in the presence of ${ }^{35} \mathrm{~S}$-UTP. The radioactive labeled riboprobes were dissolved in a hybridization 
mixture, and deposited on the sections. The hybridization $\left(16 \mathrm{~h}\right.$ at $\left.50^{\circ} \mathrm{C}\right)$ was followed by a series of washings at various stringencies and temperatures and one step of RNAse treatment to cleave off the single strands of the unhybridized riboprobes. Macroscopic semiquantitative evaluation of the hybridization signal was carried out by apposition of the slides on an X-ray film (Kodak XMR) which was photographically processed after $48 \mathrm{~h}$. This macroscopic picture on the film revealed an overview of the tissue distribution of the labeling with a view of the whole section at once. A microscopic image was obtained on the same slides as the macroscopic pictures after dipping in liquid photographic emulsion (NTB2 Kodak). The microscopic pictures were obtained after photographic development and counterstaining with toluidine blue. These slides were used to evaluate the distribution of the labeled cells and to compare the intensity of the hybridization signals between the different cancers at increasing stages of tumor progression. The following cDNAs were used to generate cRNA labeled probes: PDGF-R $\beta$ (920 bp); VEGF-A (576 bp); VEGF-R1 (1,610 bp) and VEGF-R2 (1,350 bp); NRP-1 (1,929 bp) and NRP-2 (2,102 bp); EphrinB1 (941 bp) and Ephrin B2 (1,069 bp); EPHB4 (972 bp); Net-1 (1,900 bp); AGT (1,000 bp); TSP$2(1,380 \mathrm{bp})$. In parallel and in the same series of slides, control of labeling specificity was carried out using the sense probes. No specific localization was observed; only a weak and uniform low-level background could be detected (results not shown).

\section{Evaluation of hybridized slides}

The hybridized samples were quoted by three scientists (M.C., J.-M.G., and L.J.-J.), and quotations further validated independently by two pathologists (R.C.J. and B.L.). Each hybridized slide was graded following a scale between 0 (no labeling detectable) and 4 (maximum intensity of labeling). All slides of one tumor type were hybridized at the same time and with the same batch of riboprobe. In each group the individual scores of all tissue samples were averaged to obtain mean values. Since for each marker all tumors of all grades were hybridized at the same time and with the same probe batch, comparison between these samples is acceptable. These values were compared with each other within a group of sections hybridized with the same batch of radiolabeled probe.

\section{Results}

The expression of several markers was heterogeneous between tumors of the same grade. In the figures we present a consensus analysis of representative pictures.
Therefore, in this manuscript "representative" means that the tumor tissue examined displayed the histological characteristics of the type of cancer and the grade defined, and corresponded to the majority of the messenger RNA (mRNA) expression analyzed. However, there may be minor areas of a specific sample, with all the requested histological characteristics, which may have a different pattern of expression. Following this evaluation process, representative pictures of the observations of primary human astroglial (Fig. 1), oligodendroglial (not shown), and ependymomal (Fig. 2) tumors allowed determination of the cells expressing the mRNAs for the selected factors. The semiquantitative mRNA expression levels are reported for tumoral tissue (Table 1) and its associated vasculature (Table 2).

Astroglial tumors (Fig. 1) In low-grade (grade I-III) astrocytomas, VEGF-A expression was detected in a few large cells (Fig. 1, arrow) of the tumor. In high-grade tumors [grade IV astrocytomas/glioblastoma (GBM)] VEGF-A expression was highly increased in the tumor. The expression of VEGF-R1 increased with tumor grade in the tumor vasculature, in particular in the glomeruloid structures of GBM (Fig. 1, arrow). VEGF-R2 (not shown) expression was found in a few vascular and perivascular cells localized in tumors of all grades, not obviously associated with grade. NRP-1 was highly expressed by a few localized large cells in low-grade tumors, and highly expressed in some highly vascularized abnormal structures of GBM. NRP-2 expression was associated with large cells, which we identified as reactive astrocytes, in low-grade cancer, and decreased with tumor grade. PDGF-R $\beta$ expression was detected in scattered perivascular cells in low-grade astrocytomas and in the glomeruloid vessels of GBM (Fig. 1, arrow), but not in all vessels of high-grade astrocytomas. Net-1 expression (not shown) was analyzed only in low-grade astrocytoma, and was found at high level in reactive astrocytes. EphrinB1 (not shown) was expressed at low level in only a few perivascular cells in all grades of astrocytoma without obvious relationship with grade. EphrinB2 (not shown) had the highest expression of all EphB mRNAs evaluated and was mainly observed in the tumor vasculature, possibly endothelial cells, with no obvious relationship with tumor grade. EPHB4 (not shown) expression was very low in all grades of astrocytoma. In agreement with the our previous results [5], AGT expression (not shown) was mainly localized in large cells, decreasing with the increase of tumor grade. The level of TSP-2 expression did not markedly change with grade, but the type of cells expressing it was modified. TSP-2 was expressed by large perivascular cells in low-grade tumors (Fig. 1, arrow), and by smaller cells in high-grade astrocytomas.

Oligodendroglial tumors (not shown) VEGF-A was not, or only at very low level, expressed in low-grade (grade II) oligodendrogliomas, but dramatically increased in high- 


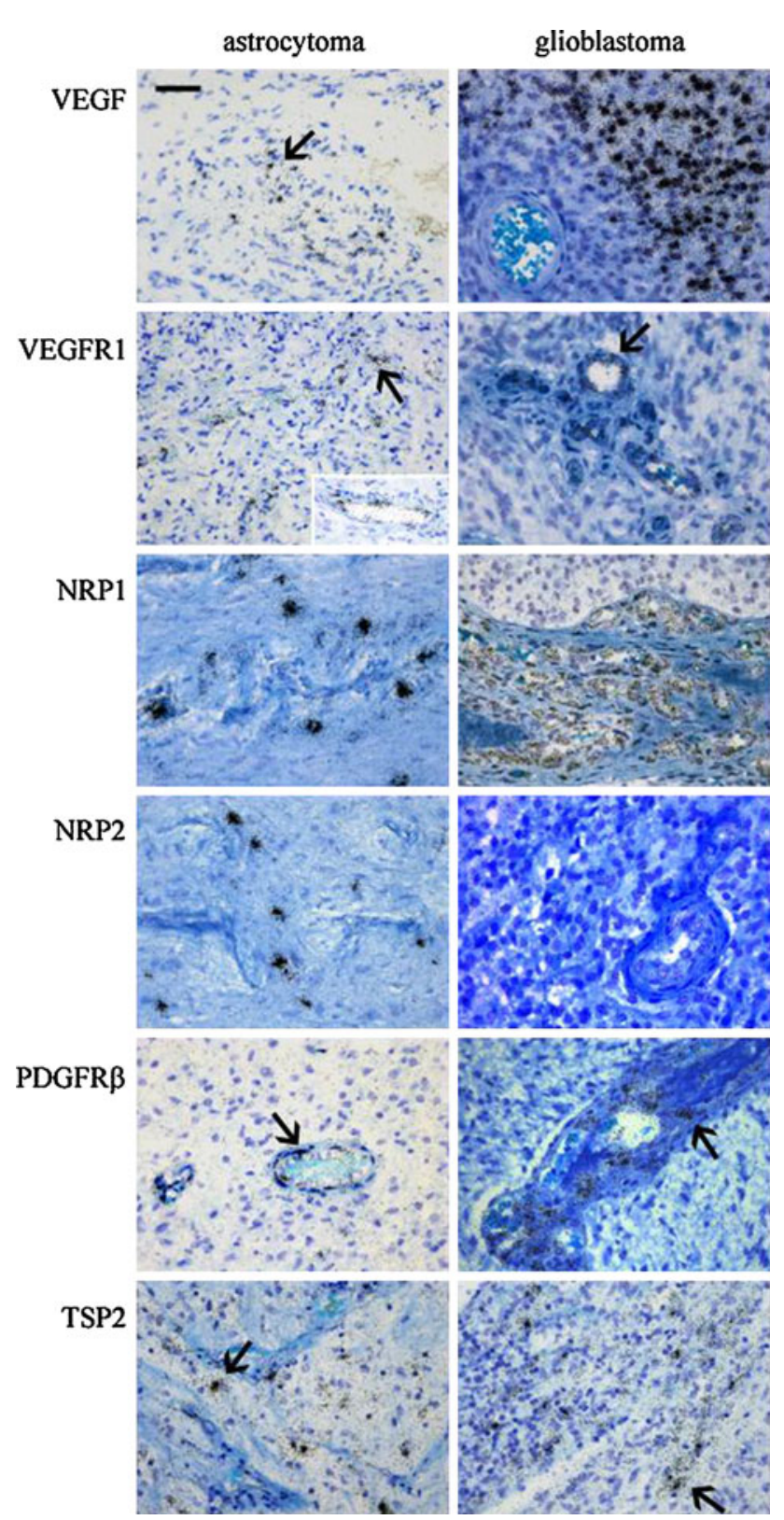

Fig. 1 Cell distribution determined by in situ hybridization of the mRNAs for neurogenic/angiogenic factors in grade I/II astrocytoma and grade IV/glioblastoma. Representative histological pictures of in situ hybridization slides of human low-grade astrocytomas (left) and high-grade astrocytoma (glioblastoma/GBM) (right) processed for in situ hybridization with the corresponding antisense cRNAs probes. Bar $50 \mu \mathrm{m}$. Arrows areas of positive labeling. Initial magnification $\times 40$

grade (grade III) tumors, whereas VEGF-R1 was already expressed in low-grade oligodendrogliomas, also increasing with grade. VEGF-R2 was not detected in low-grade oligodendrogliomas and was not evaluated in high-grade tumors. NRP-1 was expressed at higher level in nontumoral tissue surrounding the tumor than by the tumor tissue itself. Expression was high in large cells and in perivascular cells of low-grade tumors, and labeling decreased with increasing

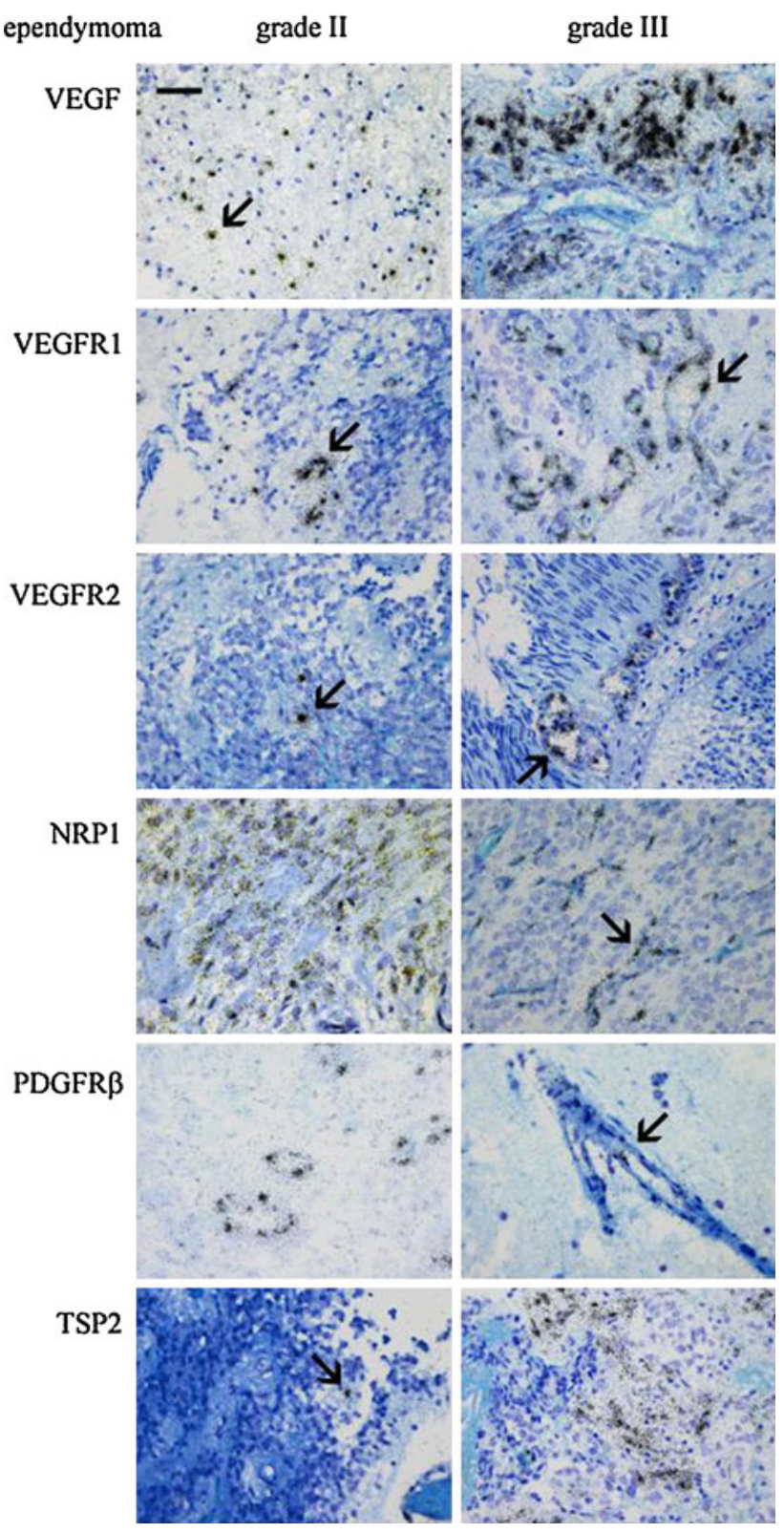

Fig. 2 Cell distribution determined by in situ hybridization of the mRNAs for neurogenic/angiogenic factors in grade II ependymoma and grade III ependymoma. Histological pictures of in situ hybridization slides of human low-grade (grade II) (left) and high-grade (grade III) (right) ependymomas processed for in situ hybridization with the corresponding antisense cRNAs probes. Bar $50 \mu \mathrm{m}$. Arrows areas of positive labeling. Initial magnification $\times 40$

tumor grade. NRP-2 was almost not expressed in all grades of cancer. In low-grade oligodendrogliomas, PDGF-R $\beta$ expression was observed in low- and high-grade oligodendrogliomas, in most tumor-associated vessels and in reactive astrocytes. Net-1 evaluation was performed only in highgrade tumors and was detected in only few scattered cells. EphrinB1, EphrinB2, and EPHB4 expression was detected in large cells of localized areas, possibly reactive astrocytes or neurons, in low-grade oligodendrogliomas, and in isolated 
Table 1 Semiquantitative evaluation of mRNA expression levels determined by in situ hybridization in the tumor tissue

\begin{tabular}{|c|c|c|c|c|c|c|c|}
\hline & astro I + II & astro III & GBM & oligo II & oligo III & epend II & epend III \\
\hline VEGF-A & + & + & +++ & $(+)$ & +++ & + & +++ \\
\hline VEGFR1 & $(+)$ & - & $(+)$ & - & - & + & + \\
\hline VEGF R2 & - & $(+)$ & - & - & n.d. & - & - \\
\hline NRP-1 & + & + & ++ & + & + & + & ++ \\
\hline NRP-2 & + & + & $(+)$ & - & - & - & $(+)$ \\
\hline PDGF-R $\beta$ & - & - & - & $(+)$ & $(+)$ & $(+)$ & - \\
\hline Net-1 & +++ & $(+)$ & + & n.d. & $(+)$ & + & + \\
\hline EphrinB1 & $(+)$ & + & + & $(+)$ & + & $(+)$ & - \\
\hline EphrinB2 & $(+)$ & + & - & $(+)$ & + & $(+)$ & - \\
\hline EPHB4 & - & + & - & $(+)$ & + & + & $(+)$ \\
\hline AGT & + & ++ & + & + & + & ++ & + \\
\hline TSP-2 & ++ & + & + & n.d. & n.d. & $(+)$ & +++ \\
\hline
\end{tabular}

- No expression; $(+),+,++,+++$ very low, low, medium and very high expression; $n . d$. not determined

Table 2 Semiquantitative evaluation of mRNA expression levels determined by in situ hybridization in the tumor-associated vasculature

\begin{tabular}{|c|c|c|c|c|c|c|c|}
\hline & astro I+II & astro III & GBM & oligo II & oligo III & epend II & epend III \\
\hline VEGF-A & + & - & - & - & - & ++ & +++ \\
\hline VEGF R1 & + & + & +++ & $(+)$ & + & ++ & +++ \\
\hline VEGF R2 & $(+)$ & $(+)$ & $(+)$ & $(+)$ & n.d. & + & ++ \\
\hline NRP-1 & + & + & ++ & - & $(+)$ & + & ++ \\
\hline NRP-2 & $(+)$ & + & $(+)$ & - & - & - & - \\
\hline PDGF-R $\beta$ & ++ & ++ & + & + & + & ++ & + \\
\hline Net-1 & ++ & - & - & n.d. & - & - & $(+)$ \\
\hline EphrinB1 & $(+)$ & $(+)$ & $(+)$ & - & - & - & - \\
\hline EphrinB2 & + & $(+)$ & + & $(+)$ & + & - & + \\
\hline ЕРHB4 & $(+)$ & $(+)$ & - & - & $(+)$ & - & $(+)$ \\
\hline AGT & + & + & - & - & + & - & - \\
\hline TSP-2 & + & + & + & n.d. & n.d. & + & + \\
\hline
\end{tabular}

- No expression; $(+),+,++,+++$ very low, low, medium, and very high expression; $n . d$. not determined

smaller cells of high-grade tumors, EphrinB2 being predominantly expressed compared with EphrinB1 and EPHB4. AGT was diffusely detected in localized cells in tumors of both grades, possibly at higher level in low-grade oligodendrogliomas. TSP-2 evaluation was not performed for oligodendrogliomas.

Ependymomal tumors (Fig. 2) VEGF-A expression was observed in scattered tumor cells of low-grade (grade II) ependymomas (Fig. 2, arrow) and at much higher levels in tumor cells surrounding tumor-associated vessels of highgrade (grade III) ependymomas, as well as the vascular cells themselves (not shown). VEGF-R1 expression was localized in the tumor vessels, and expression levels increased from low grade to high grade, associated to an increased number of vessels. The expression of VEGF-R2 (Fig. 2, arrow) was lower than that of VEGF-R1, increasing with tumor grade. The expression of NRP-1 in the tumor tissue of low-grade tumors (Fig. 2, arrow) was associated with the tumor cells rather than with the tumor vessels. NRP-1 expression increased with grade in the tumor vessels. NRP-2 (not shown) was expressed at very low level only by very few cells in both grades of tumor. In low-grade ependymomas, $\mathrm{PDGF}-\mathrm{R} \beta$ expression was observed in most tumor-associated vessels, but only in very few vascular cells of the typical "hen-feet" tumor vessels (Fig. 2, arrow) of high-grade ependymomas. Net-1 (not shown) was largely expressed by tumor cells without any obvious changes with tumor grade. In low-grade ependymomas, EphrinB1 and EPHB4 (not shown) were generally diffusely expressed at low level. EphrinB2 was expressed by the tumor vasculature in both grades of tumor. AGT (not shown) was diffusely expressed in both grades of ependymoma, without obvious relationship with grade. In lowgrade ependymomas, TSP-2 expression (Fig. 2, arrow) was observed in few isolated tumor cells and in more cells, vascular and tumor cells, of high-grade ependymomas. 
Thus, in the tumor tissue (Table 1), VEGF-A expression increased with grade, in all tumor types. However, both VEGF-R1 and VEGF-R2 were only marginally expressed. NRP-1 was expressed at low levels in low-grade tumors of the three types, and increased with tumor grade in astrocytomas. In the other cancers, NRP-2 was only marginally expressed, and its expression was not grade related. No PDGF-R $\beta$ expression was detected in the tumor tissue of all tumor types and grades. All components of the ephrinB system evaluated were only sporadically expressed, and no obvious changes of expression with grade were apparent in the tumor tissue. Confirming our previous results in astrocytomas only [5], AGT expression decreased with grade in astrocytoma and ependymoma, whereas this relation was less clear in oligodendroglioma. TSP-2 and Net-1 expression decreased with grade in astrocytomas, whereas TSP-2 expression, but not Net-1 expression, increased with tumor grade in ependymomas.

In tumor-associated vasculature (Table 2), VEGF-A was not expressed in tumor vessels in astrocytomas and oligodendrogliomas, but was highly expressed in ependymomas, increasing with tumor grade, suggesting an autocrine effect. VEGF-R1 and NRP-1 expression in tumor vessels increased with grade in astrocytomas and ependymomas, whereas VEGF-R2 expression increased with grade in ependymomas, but not in the other two types of cancers. NRP-2 was only marginally detected in all cancers of any grade. PDGF-R $\beta$ expression decreased with increasing tumor grade in astrocytomas and ependymomas. Net-1 expression was clearly observed only in low-grade astrocytoma vessels. All components of the ephrinB system evaluated were only marginally expressed in tumor-associated vessels, and no obvious changes of expression with grade were apparent. AGT was generally not expressed in all tumor-associated vessels, whereas TSP-2 expression in the tumor vessels was low and not grade related in astrocytomas, while it increased with grade in ependymomas.

\section{Discussion}

Brain tumors are highly vascularized, and the cancerassociated vasculature recapitulates in part the properties of the developmental vascularization, displaying branching structures, high permeability, and high proliferative rates. The development of the vertebrate vascular system requires precise control of the growth and branching of new vessels mediated by families of peptides and their receptors (reviewed in 1). Some of these peptide pathways have also been involved in controlling neuronal guidance. During developmental vascularization the VEGF/VEGFR/NRP system is involved in the early steps of angiogenesis, whereas the ephrin system, in particular EPHB4/ephrinB2, controls branching, maturation, and the arteriovenous asymmetry of the vasculature in later stages [7-10], while in the nervous system, the ephrin system exerts repulsive action. The VEGF-A and its receptors VEGF-R1 and VEGF-R2 are the major system shaping vasculogenesis and angiogenesis during development. NRP-1 and NRP-2 receptors can bind VEGF, forming complexes with either VEGF-R1 or VEGF-R2. NRP-1 and NRP-2 are membranebound receptors with two different ligands, the semaphorins (repulsive for axonal guidance) and the VEGFs (attractive for angiogenesis). The netrins are a group of angiogenic factors also involved in neuron migration, whose role in angiogenesis is ambiguous as it induces context-dependent responses, either pro- or antiangiogenic or attractive or repulsive effects (reviewed in 1,2). The cooperation of $\mathrm{PDGF}$ and its $\mathrm{PDGF}-\mathrm{R} \beta$ receptor is required to attract pericytes and form and stabilize fully differentiated vessels [11]. During development of the neural tube, netrin-1 antagonizes the chemoattraction of PDGF and thus prevents the effects of PDGF on the normal dispersion of precursor cells [12]. It has been previously shown in experimental models that Net-1 binding to its UNC5B receptor induces endothelial repulsion and inhibits sprouting angiogenesis [13]. The absence of the ligand Net-1 on its receptors has been shown to result in the activation of cell death by apoptosis [14]. However, these results contradict previous information which suggests that the netrins stimulate proliferation, migration, and tube formation of human endothelial cells in a UNC5B-independent manner [15].

To control vascular development, the proangiogenic pathways can be antagonized by the antiangiogenic systems. AGT, and TSP-1 and TSP-2 have been previously suggested to play antiangiogenic roles. AGT, the major source of angiotensin II, not only controls vascular wall resistance but also angiogenesis. AGT is abundantly expressed in some areas of the brain, and its antiangiogenic properties have been recently demonstrated in a mouse model overexpressing AGT [16]. TSP-1 and TSP-2 are powerful antiangiogenic factors, counterbalancing the effects of VEGF and, at least in humans, may participate in the blockade of the remodeling of a functional vascular architecture. TSP-1 and TSP-2 appear to mediate their antiantiangiogenic effects by inhibiting neoangiogenesis and endothelial cell proliferation and migration.

In cancer, VEGF-A produced by the tumor stimulates permeabilization of preexisting capillaries and proliferation of endothelial cells through paracrine activation of one of its receptors on tumor vessels, to form neovessels with distorted structures. As expected from previous information, the expression of VEGF-A mRNA increased strongly with tumor progression in the tumoral tissue, localized in clusters of tumor cells at the invasion front of the tumor. Surprisingly, the expression of not only VEGF-A but also 
its VEGF-R1 receptor by the tumor vessels increased in grade III ependymoma, suggesting an autocrine loop. In the brain cancers that we analyzed, NRP-1 and NRP-2 displayed a constant low level of expression, mainly detected in endothelial cells and in reactive perivascular cells in infiltrative areas of the tumor. It has been previously shown that NRP1 is mainly expressed by carcinomas and NRP2 mainly by neuronal tumors, glioblastomas, and melanomas [17]. In our series of human brain tumors, expression of PDGF-R $\beta$ did not increase concomitantly with the large increase of VEGF-A expression in stromal cells and VEGF-R1 in the vessel wall as tumor grade increased. Thus, we can hypothesize that, in human brain cancer, when stimulated by VEGF-A, new vessels form, but fail to organize functional vessels in the absence of PDGF-R $\beta$. The comparison between VEGF-R1 and VEGF$\mathrm{R} 2$ expression suggested that VEGF-R1 mRNA increased in the vessels with tumor progression, while VEGF-R2 decreased. Presently, we have no explanation for this observation.

The ephrin systems have been generally associated with cancer, in cancer cells and stroma, but can be downregulated at advanced stages, receptors and ligands being discordantly regulated [18]. In the primary human brain cancers that we studied, the expression of the components of the ephrinB system evaluated did not display a clear relationship with either grade or cancer type, EphrinB2 being the most highly expressed, but anyhow at low level and possibly only in the remaining nontumoral neural cells, reactive astrocytes or remaining neurons, of the cancer tissue. EPHB4 and EphrinB2 are necessary for vascular development, and for tumor angiogenesis, cross-talking with the VEGF system [1, 3, 19], however the role of EphrinB2 and EPHB4 in cancer cells is complex, paradoxical, and sometimes opposite. It has been shown that EPHB4 on tumor cells interact with EphrinB2 on endothelial cells, promoting angiogenesis, while EPHB4 may be important for tumor cell growth, either increasing or decreasing tumor growth depending on the context; for example, human colorectal cancers lose expression of EphB at the adenoma-carcinoma transition, correlated with cancer progression [20].

We show a clear decrease with tumor grade of Net-1 in the tumor tissue of astrocytomas. Net-1 was shown to be upregulated in aggressive neuroblastoma, and has been associated with poor prognosis in several other aggressive human cancers. By immunohistochemistry, Net-1 has been previously demonstrated in tumor cells, but not in the tumor stroma, confirming our present results. In experimental models of breast cancer, Net-1 binding to its receptor(s) induced migration, proliferation, and differentiation, while in colorectal cancer, loss of function of the receptors was prevalent over gain of Net-1 [21, 22].
Generally we observed a decrease of AGT expression with increasing grade of all three cancer types, confirming our previous results in astrocytomas [5]. The decrease in AGT expression corresponds to a loss of AGT antiangiogenic effects as the tumors progress. The downregulation of TSP-1 has already been documented [23], and we did not include it in our study. In our series of human primary tumors of the brain, we found that, whereas TSP-2 expression did not markedly change with tumor grade, the type of cells expressing it did change. In low-grade tumors, remaining neurons or reactive astrocytes expressed it, while it was expressed by small cells in higher-grade tumors. Using immunohistochemistry, TSP-1 and TSP-2 protein expression was previously shown to be higher in glioblastoma than in lower-grade astrocytoma, predominantly localized in vascular cells, with minimal expression in the tumor tissue [24-27]. Our results did not confirm the previously published information that TSP-2, but not TSP1 , mRNA was correlated with high grade in astrocytomas [28]. This is an important consideration when evaluating potential therapeutic targets and may explain discrepancies between series of samples.

Acknowledgments We thank Dr. P. Corvol for his continuous support, help, and constructive discussions and comments, and S. Gros, S. Trepey, and M. Lesage for excellent technical assistance. We are particularly thankful to Dr. Christine Hubert (INSERM U833) for the gift of the ephrins (B1, B2, and B4) and PDGF-R $\beta$ cDNAs, Dr. Anne Eichmann (INSERM U833) for the gift of NRP1, NRP2, and Netrin-1 cDNAs, Dr. Judith Favier (INSERM U833) for the gift of VEGF-A, VEGF-R1, and VEGF-R2 cDNAs, Dr. Eric Clauser (INSERM U36) for the gift of the AGT cDNA, and Dr. Paul Bornstein (Department of Biochemistry and Medicine, University of Washington, Seattle) for the gift of TSP-2 cDNA. This work was supported by grants of Collège-de-France (to MC) and Swiss Society for Multiple Sclerosis (to L.J.-J.).

\section{References}

1. Raab S, Plate KH (2007) Different networks, common growth factors: shared growth factors and receptors of the vascular and the nervous system. Acta Neuropathol 113:607-626

2. Larivée B, Freitas C, Suchting S, Brunet I, Eichmann A (2009) Guidance of vascular development. Lessons from the nervous system. Circ Res 104:428-441

3. Suchting S, Bicknell R, Eichmann A (2006) Neuronal clues to vascular guidance. Exp Cell Res 312:668-678

4. Plate KH, Warnke PC (1997) Vascular endothelial growth factor. J Neurooncol 35:365-372

5. Juillerat-Jeanneret L, Célérier J, Chapuis Bernasconi C, Nguyen G, Wostl W, Maerki HP, Janzer RC, Corvol P, Gasc JM (2004) Renin and angiotensinogen expression and function in growth and apoptosis of human glioblastoma. Br J Cancer 90:1059-1068

6. Sibony M, Commo F, Callard P, Gasc JM (1995) Enhancement of mRNA in situ hybridization signal by microwave heating. Lab Invest 73:586-591

7. Sawamiphak S, Seidel S, Essmann CL, Wilkinson GA, Pitulescu ME, Acker T, Acker-Palmer A (2010) Ephrin-B2 regulates 
VEGFR2 function in developmental and tumour angiogenesis. Nature 465:487-491

8. Wang Y, Nakayama M, Pitulescu ME, Schmidt TS, Bochenek ML, Sakakibara A, Adams S, Davy A, Deutsch U, Lüthi U, Barberis A, Benjamin LE, Mäkinen T, Nobes CD, Adams RH (2010) Ephrin-B2 controls VEGF-induced angiogenesis and lymphangiogenesis. Nature 465:483-486

9. Germain S, Eichmann A (2010) VEGF and ephrinB2: a bloody duo. Nat Med 16:752-754

10. Héroult M, Schaffner F, Augustin HG (2006) Eph receptor and ephrin ligand-mediated interactions during angiogenesis and tumor progression. Exp Cell Res 312:642-650

11. Zhang J, Cao R, Zhang Y, Jia T, Cao Y, Wahlberg E (2009) Differential roles of PDGFR- $\alpha$ and PDGFR- $\beta$ in angiogenesis and vessel stability. FASEB J 23:153-163

12. Tsai HH, Tessier-Lavigne M, Miller RH (2003) Netrin 1 mediates spinal cord oligodendrocyte percussor dispersal. Development 130:2095-2105

13. Larivée B, Freitas C, Trombe M, Ly X, DeLafarge B, Yuan L, Bouvrée K, Bréant C, Del Toro R, Bréchot N, Germain S, Bono F, Dol F, Claes F, Fischer C, Autiero M, Thomas JL, Carmeliet P, Tessier-Lavigne M, Eichmann A (2007) Activation of the UNC5B receptor by netrin-1 inhibits sprouting angiogenesis. Genes Dev 21:2433-2447

14. Bernet A, Fitamant J (2008) Netrin-1 and its receptors in tumour growth promotion. Expert Opin Ther Targets 12:995-1007

15. Wilson BD, Li M, Park KW, Suli A, Sorensen LK, LarrieuLahargue F, Urness LD, Suh W, Asai J, Kock GAH, Thorne T, Silver M, Thomas KR, Chien CB, Losordo DW, Li DY (2006) Netrins promote developmental and therapeutic angiogenesis. Science 313:640-644

16. Vincent $F$, Bonnin $P$, Clemessy M, Contrerès JO, Lamandé N, Gasc JM, Vilar J, Hainaud P, Tobelem G, Corvol P, Dupuy E (2009) Angiotensinogen delays angiogenesis and tumour growth of hepatocarcinoma in transgenic mice. Cancer Res b69:2853-2860

17. Bielenberg DR, Pettaway CA, Takashima S, Klagsbrun M (2006) Neuropilins in neoplasms: expression, regulation and function. Exp Cell Res 312:584-593

18. Pasquale EB (2010) Eph receptors and ephrins in cancer: bidirectional signalling and beyond. Nat Rev Cancer 10:165-180
19. Martiny-Baron G, Holzer P, Schnell C, Brueggen J, Ferretti M, Schmiedberg N, Wood JM, Furet P, Imbach P (2010) The small molecule specific EphB4 kinase inhibitor NVP-BHG712 inhibits VEGF-driven angiogenesis. Angiogenesis 13:259-267

20. Battle E, Bacani J, Begthel H, Jonkeer S, Gregorieff A, van de Born M, Malats N, Sancho E, Boon E, Gallinger S, Pals S, Clevers H (2005) EphB receptor activity suppresses colorectal cancer progression. Nature 435:1126-1130

21. Delloye-Bourgeois C, Fitamant J, Paradisi A, Capellen D, DoucRasy S, Raquin MA, Stupack D, Nakagarawa A, Rousseau R, Combaret V, Puisieux VA, Valteau-Couanet D, Bénard J, Bernet A, Mehlen P (2009) Netrin-1 acts as a survival factor for aggressive neuroblastoma. J Exp Med 206:833-847

22. Mehlen P, Guenebaud C (2010) Netrin-1 and its dependence receptors as original targets for cancer therapy. Curr Opin Oncol 22:46-54

23. Tenan M, Fulci G, Albertoni M, Diserens AC, Hamou MF, El AtifiBorel M, Feige JJ, Pepper MS, Van Meir EG (2000) Thrombospondin-1 is downregulated by anoxia and suppresses tumorigenicity of human glioblastoma cells. J Exp Med 191:1789-1798

24. Rege TA, Fears CY, Gladson CL (2005) Endogenous inhibitors of angiogenesis in malignant gliomas: nature's antiangiogenic therapy. Neuro Oncol 7:106-121

25. Pijuan-Thompson V, Grammer JR, Stewart J, Silverstein RL, Pearce SF, Tuszynski GP, Murphy-Ullrich JE, Gladson CL (1999) Retinoic acid alters the mechanism of attachment of malignant astrocytoma and neuroblastoma cells to thrombospondin-1. Exp Cell Res 249:86-101

26. Hsu SC, Volpert OV, Steck PA, Mikkelsen T, Polverini PJ, Rao S, Chou P, Bouck NP (1996) Inhibition of angiogenesis in human glioblastomas by chromosome 10 induction of thrombospondin1. Cancer Res 56:5684-5691

27. Kawataki T, Naganuma H, Sasaki A, Yoshikawa H, Tasaka K, Nukui H (2000) Correlation of thrombospondin-1 and transforming growth factor-beta expression with malignancy of glioma. Neuropathology 20:161-169

28. Kazuno M, Tokunaga T, Oshika Y, Tanaka Y, Tsugane R, Kijima H, Yamazaki H, Ueyama Y, Nakamura M (1999) Thrombospondin-2 (TSP2) expression is inversely correlated with vascularity in glioma. Eur J Cancer 35:502-506 